\title{
Epidemiological studies of oats consumption and risk of cancer and overall mortality
}

\author{
Paolo Boffetta $^{1 *}$, Frank Thies ${ }^{2}$ and Penny Kris-Etherton ${ }^{3}$ \\ ${ }^{1}$ The Tisch Cancer Institute and Institute for Translational Epidemiology, Icabn School of Medicine at Mount Sinai, \\ New York, NY 10029, USA \\ ${ }^{2}$ Division of Applied Medicine, University of Aberdeen, Polwarth Building, Foresterbill, Aberdeen AB25 2ZD, Scotland, UK \\ ${ }^{3}$ Department of Nutritional Sciences, Pennsylvania State University, University Park, PA 16802, USA
}

(Submitted 3 October 2013 - Final revision received 21 May 2014 - Accepted 17 June 2014)

\section{Abstract}

A review of epidemiological studies on the intake of oats and oat-based products and its effect on the risk of chronic disease and deaths was performed. Seven studies were identified of cancer risk (two each on prostate and colorectal cancer, and one each on pancreatic, breast and endometrial cancer), and one study on overall mortality. With the exception of a case-control study of pancreatic cancer, all studies were of cohort design: five studies were based on a single cohort from Denmark. The results of most cohort studies suggest a weak protective effect of a high intake of oats on cancer risk (relative risks in the order of 0.9). Potential limitations of the studies are dietary exposure misclassification, low statistical power because of limited exposure contrast and residual confounding. Despite the evidence from experimental and mechanistic studies of a protective effect of oats intake on CVD and diabetes, no epidemiological studies have been conducted on these conditions.

Key words: Oats: Epidemiology: Neoplasm

There is strong evidence for a protective effect of oats intake on CVD - and possibly other chronic diseases - from shortterm dietary experimental and mechanistic studies in healthy volunteers, assessing cardiovascular risk factors including a decrease in serum LDL-cholesterol and blood pressure, and improvement in glucose and insulin response ${ }^{(1-3)}$.

Epidemiological studies are the best way to test the hypothesis of an association between diet and disease ${ }^{(4)}$, although their ability to assess the effects of specific food items is limited. They provide strong evidence for a protective effect of whole-grain intake against several diseases, including cancer, CVD and diabetes ${ }^{(5-7)}$. The role of specific wholegrain foods, such as oats, however, is less clear. The present study aims at reviewing the evidence from epidemiological studies on oats and oat-product intake and the risk of chronic disease and death.

\section{Methods}

Fig. 1 is a flow diagram showing how relevant articles were selected. A PubMed search was performed using the terms 'oat*' AND 'disease' OR 'cardiovascular' OR 'cancer' OR 'mortality' AND 'humans' OR 'epidemiology'. The search identified 2100 references, whose titles were individually checked, which resulted in the exclusion of 1596 references in which the term "oat"' did not refer to the cereal. Among the remaining 504 references, 399 were excluded after consideration of the abstract, because they concerned aspects of oats consumption not relevant to epidemiology (namely nutritional, animal or mechanistic studies). The remaining 105 references were reviewed in detail: six epidemiological studies were identified that reported results on disease incidence/ mortality and dietary intake of oats, and the remaining ninety-nine studies were mainly clinical and experimental studies. A review of the lists of references of the epidemiological studies led to the identification of two additional investigations.

For each epidemiological study, information on study characteristics (study design, population, methods for the assessment of dietary intake, confounder and outcome) and results was abstracted according to a standard format. Given the small number of studies for each type of cancer and the heterogeneity in the assessment of oats intake, no formal combination of results in a meta-analytic approach was performed.

Abbreviation: RR, relative risk.

*Corresponding author: P. Boffetta, email paolo.boffetta@mssm.edu 


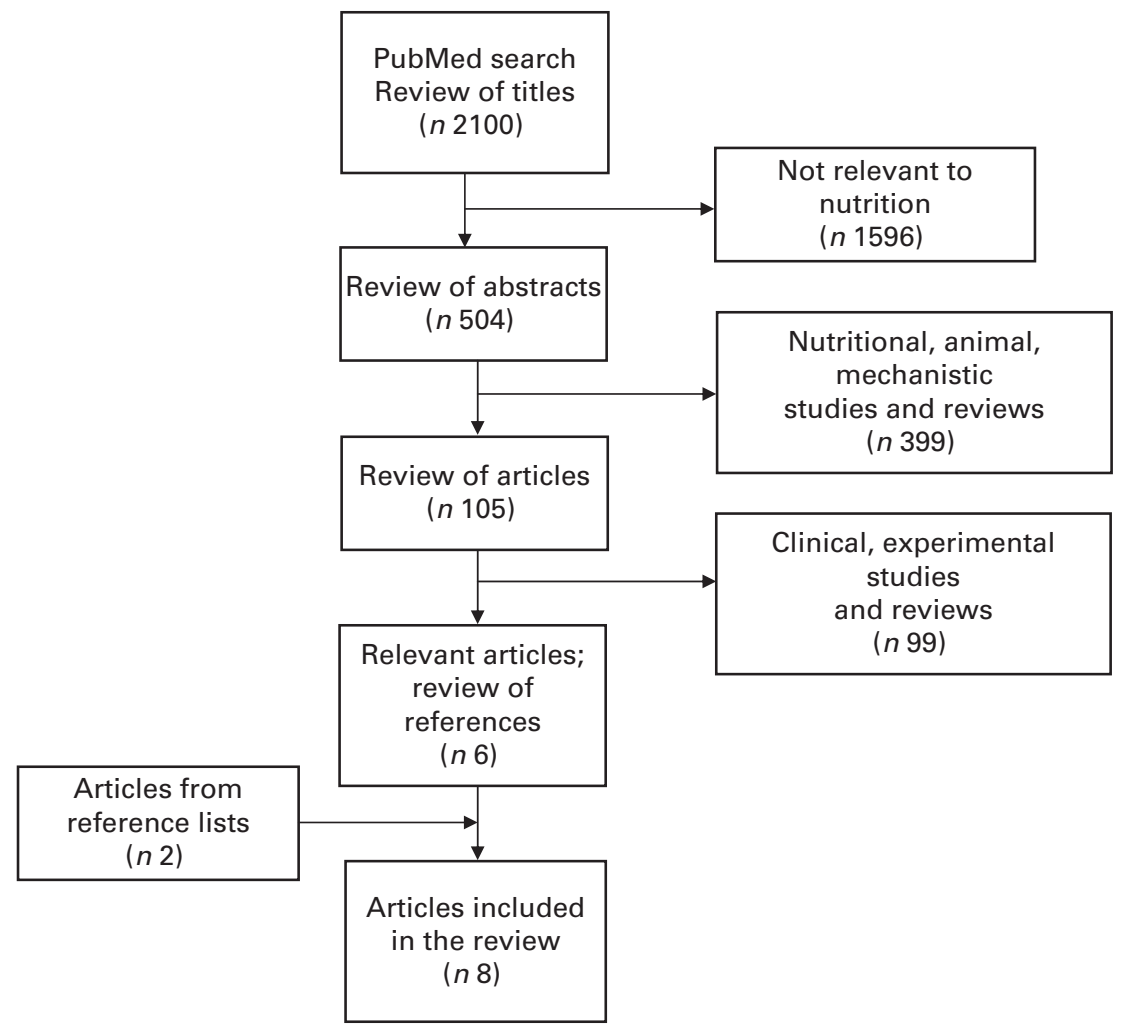

Fig. 1. Flow diagram of article selection.

\section{Results}

Seven of the studies reported results on cancer risk (two each on prostate and colorectal cancer, and one each on pancreatic, breast and endometrial cancer), and one study on overall mortality.

\section{Colorectal cancer}

In an analysis of 1025 cases of colorectal cancer diagnosed among 57053 participants in the Danish Diet, Cancer and Health cohort study over a 13-year period, no association was found with the healthy Nordic food index, which includes oatmeal intake ${ }^{(8)}$. Consumption of oatmeal was similar among those diagnosed with colorectal cancer and those who remained free of the disease.

The HELGA cohort comprises 108000 Danish, Swedish and Norwegian people (including the Danish Diet, Cancer and Health cohort), of whom 1123 developed colorectal cancer during a median of 11 years of follow-up. This cohort was analysed for the intake of whole-grain products, including whole-grain oats ${ }^{(9)}$. The intake of whole-grain products was associated with a lower incidence of colorectal cancer; however, no consistent association was observed with the intake of wholegrain oats (Table 1). The relative risk (RR) for a 10-g increase in whole-grain oats intake was 1.00 (95\% CI 0.94, 1.06).

\section{Prostate cancer}

An analysis of whole-grain product intake and the risk of prostate cancer was conducted among 26691 men aged 50-64 who participated in the Danish Diet, Cancer and Health cohort study $^{(10)}$. During a median follow-up of 12.4 years, 1081 prostate cancer cases were diagnosed in the cohort. There was no association between the total intake of wholegrain products and the prostate cancer risk $(\mathrm{RR} / 50 \mathrm{~g}$ per $\mathrm{d}$ $1.00 ; 95 \%$ CI $0.96,1.05)$. RR for an increase of $25 \mathrm{~g} / \mathrm{d}$ of oatmeal was 0.96 (95\% CI $0.88,1.06)$. In the categorical analysis, a negative association was found between the oatmeal intake and the prostate cancer risk (Table 2).

In 2002-2006, 2268 men aged 67-96 reported their dietary habits in the AGES-Reykjavik cohort study ${ }^{(11)}$. Dietary habits were assessed for early-, mid- and current life using a validated FFQ. A total of 347 participants had or were diagnosed with prostate cancer during follow-up in 2009, sixty-three of whom had an advanced form of the disease (stage $3+$ or

Table 1. Relative risk (RR) of colorectal cancer and whole-grain oats intake $^{(9)}$ Danish Diet, Cancer and Health Study

(Number of cases, relative risks and $95 \%$ confidence intervals)

\begin{tabular}{|c|c|c|c|c|}
\hline \multicolumn{2}{|c|}{ Intake (g/d) } & \multirow[b]{2}{*}{$n$} & \multirow[b]{2}{*}{$\mathrm{RR}^{*}$} & \multirow[b]{2}{*}{$95 \% \mathrm{Cl}$} \\
\hline Men & Women & & & \\
\hline $0-0.2$ & $0-0.4$ & 377 & 1.00 & Reference \\
\hline $0.3-2.0$ & $0.5-2.0$ & 237 & 0.90 & $0.76,1.06$ \\
\hline $2 \cdot 1-13 \cdot 0$ & $2 \cdot 1-7 \cdot 0$ & 232 & 0.84 & $0.71,1.05$ \\
\hline$\geq 13 \cdot 1$ & $\geq 7 \cdot 1$ & 277 & 0.88 & $0.74,1.05$ \\
\hline
\end{tabular}

*Adjusted for study centre, age, sex, alcohol intake, smoking status, education, use of hormonal replacement therapy (women only), BMI, energy intake, intake of red and processed meat, whole-grain wheat and whole-grain rye. 
Table 2. Relative risk (RR) of prostate cancer and oatmeal intake. Danish Diet, Cancer and Health study $^{(10)}$

(Number of cases, relative risks and $95 \%$ confidence intervals)

\begin{tabular}{lccc}
\hline Oatmeal intake $(\mathrm{g} / \mathrm{d})$ & $n$ & $\mathrm{RR}^{*}$ & $95 \% \mathrm{Cl}$ \\
\hline$<4$ & 624 & 1.00 & Reference \\
$4-<40$ & 210 & 0.95 & $0.77,1.18$ \\
$\geq 40$ & 247 & 0.90 & $0.73,1.11$ \\
\hline *Adjusted for age, height, weight, education, smoking status, \\
$\begin{array}{l}\text { intake of red meat, processed meat, dairy products, whole- } \\
\text { grain rye bread and whole-grain bread. }\end{array}$
\end{tabular}

died of prostate cancer). Oatmeal intake, either in adolescence or in midlife, was not associated with prostate cancer risk (Table 3). Results were similar when cases were stratified according to the disease stage.

\section{Pancreatic cancer}

Grain intake was examined in a population-based case-control study of pancreatic cancer conducted in the San Francisco Bay Area from 1995 to $1999^{(12)}$. A 131-item semiquantitative FFQ was administered to 532 cases and 1701 controls. People who consumed two or more servings of whole grains daily had a lower risk of pancreatic cancer than those who consumed less than one serving per d (OR 0.60, $95 \%$ CI $0 \cdot 31,1 \cdot 2)$. Surprisingly, the intake of cooked oatmeal or oat bran, on the other hand, was associated with a small but statistically significant increase in risk (Table 4), but this should be interpreted with caution because this may be due to recall bias.

\section{Breast cancer}

The association between the intake of whole-grain products and the risk of breast cancer was investigated among 25278 postmenopausal women participating in the Danish Diet, Cancer and Health cohort study $(1993-7)^{(13)}$. During a mean follow-up time of 9.6 years, 978 breast cancer cases were diagnosed. There was no association between the intake of wholegrain products (and specifically oatmeal) and the risk of breast cancer. For each incremental increase in the intake of total whole-grain products of $50 \mathrm{~g} / \mathrm{d}$, the adjusted RR was 1.01 (95\% CI 0.96, 1.07). The median intake of oatmeal was $7 \mathrm{~g} / \mathrm{d}$ in both cases and controls.

\section{Endometrial cancer}

The association between the intake of whole grains and the risk of endometrial cancer was investigated among 24418 women aged 50-64 at enrolment in 1993-7 in the Danish Diet, Cancer and Health cohort ${ }^{(14)}$. A total of 217 women were diagnosed with endometrial cancer during a follow-up to 2009. No association was found with either the intake of oatmeal/muesli (RR for a $50 \mathrm{~g} / \mathrm{d}$ increase 1.03; 95\% CI 0.67, 1.58) or the intake of whole oats (RR for a $5 \mathrm{~g} / \mathrm{d}$ increase $0.99 ; 95 \%$ CI $0.93,1.06$ ).

\section{Overall mortality}

Oatmeal was one of the components of the healthy Nordic food index, whose association with total mortality was investigated in the Danish Diet, Cancer and Health cohort study (57053 subjects aged 50-64 at baseline) ${ }^{(15)}$. During 12 years of follow-up, 4126 of the cohort participants died. A onepoint increase in the index score was associated with a significantly lower mortality for both men (RR 0.96; $95 \%$ CI 0.92, 0.99) and women (RR 0.96; 95\% CI 0.92, 1.00). Among the individual index components, whole-grain rye bread intake was the factor most consistently associated with lower mortality. An association was suggested for oatmeal intake: RR for $>20 v . \leq 20 \mathrm{~g} / \mathrm{d}$ was $0.91(95 \%$ CI $0.81,1.02)$ in men and 0.97 (95\% CI $0 \cdot 84,1 \cdot 11)$ in women.

\section{Discussion}

Consumption of whole grains and indices of 'healthy diet' including oats have been associated with a reduced risk of CVD, diabetes and cancer ${ }^{(5,6,16)}$, but the available evidence does not suggest a specific effect of oats and oatmeal. The available results, however, are limited and primarily based on a single cohort study from Denmark, and are limited by heterogeneity in study populations and dietary exposure assessment methods between studies. No conclusions can therefore be confidently drawn on the presence or absence of a protective effect of oats consumption on cancer risk in epidemiological studies.

Misclassification of dietary assessment is a major limitation of nutritional epidemiology studies ${ }^{(17)}$ : in prospective cohort studies, misclassification of past diet often results in the underestimation of existing associations between dietary factors and diseases. Low statistical power is an additional problem: it can not only be a result of the small number of cohort members experiencing the outcome of interest, but can also be due to the limited variability in dietary intake between study subjects. As an example, the 57053 subjects of the Danish Diet, Cancer and Health study included in the analysis of colorectal cancer

Table 3. Relative risk of prostate cancer and oatmeal intake. AGES-Reykjavik study ${ }^{(11)}$

(Odds ratios and $95 \%$ confidence intervals)

\begin{tabular}{|c|c|c|c|c|}
\hline Period of life & $\begin{array}{l}\text { Cases } \\
(n)\end{array}$ & $\begin{array}{c}\text { Non-cases } \\
(n)\end{array}$ & $\mathrm{OR}^{*}$ & $95 \% \mathrm{Cl}$ \\
\hline \multicolumn{5}{|l|}{ Adolescence } \\
\hline \multicolumn{5}{|c|}{$\begin{array}{l}\text { Frequency of intake } \\
\text { (times per week) }\end{array}$} \\
\hline $0-4$ & 203 & 1183 & 1.00 & Reference \\
\hline $5+$ & 139 & 730 & 0.99 & $0.77,1.27$ \\
\hline \multicolumn{5}{|c|}{$\begin{array}{l}\text { Midlife } \\
\text { Frequency of intake } \\
\text { (times per week) }\end{array}$} \\
\hline $0-4$ & 246 & 1441 & 1.00 & Reference \\
\hline $5+$ & 97 & 475 & $1 \cdot 10$ & $0.83,1.44$ \\
\hline
\end{tabular}


Table 4. OR of pancreatic cancer and frequency of cooked oatmeal/oat bran intake ${ }^{(12)}$

(Number of cases and controls, percentages, odds ratios and $95 \%$ confidence intervals)

\begin{tabular}{|c|c|c|c|c|c|c|}
\hline \multirow[b]{2}{*}{ Frequency of intake } & \multicolumn{2}{|c|}{ Cases } & \multicolumn{2}{|c|}{ Controls } & \multirow[b]{2}{*}{$\mathrm{OR}^{*}$} & \multirow[b]{2}{*}{$95 \% \mathrm{Cl}$} \\
\hline & $n$ & $\%$ & $n$ & $\%$ & & \\
\hline$<1$ time/month & 235 & 45 & 851 & 50 & 1.0 & Reference \\
\hline 1 time/month-1 time/week & 155 & 29 & 491 & 29 & $1 \cdot 2$ & $0.94,1.5$ \\
\hline$\geq 2$ times/week & 136 & 26 & 359 & 21 & $1 \cdot 3$ & $1 \cdot 0,1 \cdot 7$ \\
\hline
\end{tabular}

* Test for linear trend, $P$-value $=0.02$.

†Adjusted for age, sex, total energy intake, BMI, race, education, smoking and history of diabetes.

had a median intake of oatmeal equal to just $1 \mathrm{~g} / \mathrm{d}^{(8)}$ : in other words, a large proportion of this cohort provided no information relevant to the study's hypothesis.

Confounding is a further potential limitation of epidemiological studies on oats intake and disease. This arises when some risk factor of the disease is correlated with the particular exposure that is of interest and is responsible for the apparent association between the exposure of interest and the disease. For example, smokers might have a lower intake of oats compared to non-smokers, and smoking is causally associated with several cancers: this would generate an apparent inverse association between oats intake and cancer risk. Confounding can be controlled for in the statistical analysis (in the earlier example, the association between oats and cancer would be assessed separately in smokers and in non-smokers); for a proper control of confounding, however, all potential confounders should be identified and precisely measured. This is difficult to achieve in nutritional epidemiology, because of the multitude of (dietary and non-dietary) factors that could be acting as confounders, and residual confounding might still be present despite the efforts of the investigators to control for it. In most of the studies included in the present review, there was an apparent protective association between the intake of oats and oat-based products and disease in unadjusted analyses, but this became weaker after adjusting for potential dietary and non-dietary confounders, including total energy intake, intake of other whole-grain foods, BMI, tobacco smoking and education.

Fig. 2 illustrates the confounding exerted by lifestyle factors on the association between oatmeal intake and overall mortality in the Danish Diet, Cancer and Health cohort ${ }^{(15)}$ : in both men and women there was a strong, statistically significant protective effect of high oatmeal intake, but this was reduced by two-thirds in men and by half in women (in both sexes, losing statistical significance) after adjustment for a number of lifestyle and nutritional factors. It is unclear whether adjustment for additional dietary or non-dietary variables would have resulted in further attenuation of the association between oatmeal intake and mortality.

Despite these limitations, and the lack of statistical significance of adjusted results, the results of most cohort studies do suggest a weak protective effect of a high intake of oats on cancer risk (RR in the order of 0.9). The results of the single case-control study ${ }^{(12)}$, suggestive of an increased risk for high oats intake, should be interpreted with caution given the specific limitations of the present study design, notably the possibility of recall bias (i.e. the presence of the disease affecting the way in which people self-report on their diet $)^{(18)}$.

The strongest experimental evidence for the protective effect of oats is in CVD and diabetes ${ }^{(1,2,5)}$ : as large-scale epidemiological studies with the relevant data exist (e.g. NIH-AARP ${ }^{(19)}$ ), specific analyses should be performed on the impact of oats
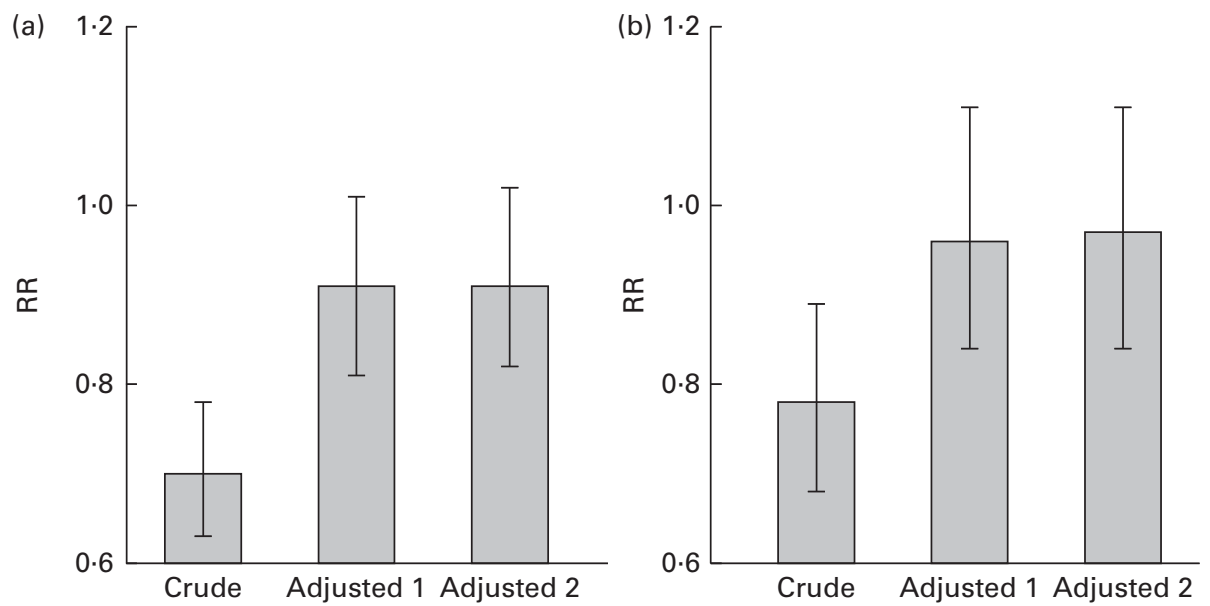

Fig. 2. Crude and adjusted relative risk (RR) of overall mortality for oatmeal intake ${ }^{(15)}$ of $>20 \mathrm{v}$. $\leq 20 \mathrm{~g} / \mathrm{d}$ in (a) men and (b) women. Values are relative risk and $95 \%$ confidence intervals represented by vertical bars. Crude: adjusted only for time. Adjusted 1: additional adjustment for tobacco smoking, alcohol drinking, education, physical activity, BMI, intake of red meat, processed meat and total energy. Adjusted 2: additional adjustment for the intake of fish, cabbages, apples and pears, rye bread and root vegetables. 
consumption on the risk of these diseases, as well as cancer. Such analyses could combine data on oatmeal and oatcontaining breakfast cereals: although they might not be considered definitive, they would provide relevant information and would show the difficulty in studying the disease risk in rarely eaten foods.

\section{Conclusion}

In conclusion, few epidemiological studies have been conducted on oats and oat-based products and disease risk: overall, they provide weak evidence of a protective effect of oats intake on cancer risk and overall mortality. Epidemiological studies on the risk of cancer, CVD and other chronic diseases such as diabetes should be conducted, based on existing large-scale cohorts with information on the intake of this group of foods.

\section{Acknowledgements}

P. B., F. T. and P. K.-E. each received an honorarium from Quaker Oats Company (a subsidiary of PepsiCo, Inc.) for attending a workshop in May 2012 to discuss the content of the supplement. P. B. (Icahn School of Medicine at Mount Sinai), F. T. (University of Aberdeen) and P. K.-E. (Pennsylvania State University) received unrestricted grants from Quaker Oats Company.

The authors' contributions are as follows: P. B., F. T. and P. K.-E. conceived the review; P. B. extracted the data and prepared the first draft of the paper. All authors reviewed and commented on the paper.

This paper was published as part of a supplement to British Journal of Nutrition, publication of which was supported by an unrestricted educational grant from Quaker Oats Co. (a subsidiary of PepsiCo Inc.). The papers included in this supplement were invited by the Guest Editor and have undergone the standard journal formal review process. They may be cited.

The Guest Editor to this supplement is Roger Clemens. The Guest Editor declares no conflict of interest.

\section{References}

1. Harris KA \& Kris-Etherton PM (2010) Effects of whole grains on coronary heart disease risk. Curr Atheroscler Rep 12, 368-376.

2. Thies F, Masson LF, Boffetta P, et al. (2014) Oats and CVD risk markers: the impact of oat and $\beta$-glucan intake. $\mathrm{Br} J$ Nutr 112, S19-S30

3. Thies F, Masson LF, Boffetta P, et al. (2014) Oats and bowel disease: a systematic literature review. Br J Nutr $\mathbf{1 1 2}$, S31-S43.
4. Willett W (2013) Overview of nutritional epidemiology. In Nutritional Epidemiology, 3rd ed., pp. 1-16 [W Willett, editor]. New York, NY: Oxford University Press.

5. Gil A, Ortega RM \& Maldonado J (2011) Wholegrain cereals and bread: a duet of the Mediterranean diet for the prevention of chronic diseases. Public Health Nutr 14, 2316-2322.

6. Fardet A (2010) New hypotheses for the health-protective mechanisms of whole-grain cereals: what is beyond fibre? Nutr Res Rev 23, 65-134.

7. Slavin J (2003) Why whole grains are protective: biological mechanisms. Proc Nutr Soc 62, 129-134.

8. Kyrø C, Skeie G, Loft S, et al. (2013) Adherence to a healthy Nordic food index is associated with a lower incidence of colorectal cancer in women: the Diet, Cancer and Health cohort study. Br J Nutr 109, 920-927.

9. Kyrø C, Skeie G, Loft S, et al. (2013) Intake of whole grains from different cereal and food sources and incidence of colorectal cancer in the Scandinavian HELGA cohort. Cancer Causes Control 24, 1363-1374.

10. Egeberg R, Olsen A, Christensen J, et al. (2011) Intake of whole-grain products and risk of prostate cancer among men in the Danish Diet, Cancer and Health cohort study. Cancer Causes Control 22, 1133-1139.

11. Torfadottir JE, Valdimarsdottir UA, Mucci L, et al. (2012) Rye bread consumption in early life and reduced risk of advanced prostate cancer. Cancer Causes Control 23, 941-950.

12. Chan JM, Wang F \& Holly EA (2007) Whole grains and risk of pancreatic cancer in a large population-based case-control study in the San Francisco Bay Area, California. Am J Epidemiol 166, 1174-1185.

13. Egeberg R, Olsen A, Loft S, et al. (2009) Intake of whole grain products and risk of breast cancer by hormone receptor status and histology among postmenopausal women. Int J Cancer 124, 745-750.

14. Aarestrup J, Kyrø C, Christensen J, et al. (2012) Whole grain, dietary fiber, and incidence of endometrial cancer in a Danish cohort study. Nutr Cancer 64, 1160-1168.

15. Olsen A, Egeberg R, Halkjær J, et al. (2011) Healthy aspects of the Nordic diet are related to lower total mortality. J Nutr 141, 639-644.

16. Haas P, Machado MJ, Anton AA, et al. (2009) Effectiveness of whole grain consumption in the prevention of colorectal cancer: meta-analysis of cohort studies. Int J Food Sci Nutr 60, Suppl. 6, 1-13.

17. Willett W \& Lenart E (2013) Reproducibility and validity of food frequency questionnaires. In Nutritional Epidemiology, 3rd ed., pp. 96-141 [W Willett, editor]. New York, NY: Oxford University Press.

18. Willett W (2013) Recall of remote diet. In Nutritional Epidemiology, 3rd ed., pp. 142-149 [W Willett, editor]. New York, NY: Oxford University Press.

19. Schatzkin A, Mouw T, Park Y, et al. (2007) Dietary fiber and whole-grain consumption in relation to colorectal cancer in the NIH-AARP Diet and Health Study. Am J Clin Nutr 85, $1353-1360$. 